\title{
A three dimensional smooth particle hydrodynamics model of the nanoscale condensation of water.
}

\author{
$\underline{\text { A. N. Charles }}^{\text {a }}$, P. Daivis ${ }^{\mathrm{a}}$

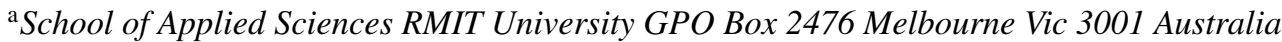 \\ Email: a.charles@bom.gov.au
}

\begin{abstract}
Mesoscopic, coarse grained models of phase transitions are of interest for their potential to simulate the transient fine scale structure associated with rapid phase transitions and the equilibrium properties of multi-phase systems. The van der Waals square gradient model provides a convenient equation of state for a fluid with a liquid and vapour phase. A three dimensional smooth particle hydrodynamics code was developed in order to investigate the fidelity with which this numerical technique captures the behaviour of the van der Waals model, parameterised for water in conditions of liquid-vapour coexistence. Our code is capable of modelling small to medium sized systems of the order of several thousands of particles in three dimensions, at scales of 10-100 nanometres.
\end{abstract}

Lagrangian methods such as smooth particle hydrodynamics (SPH) are capable of simulating flows with complex structure naturally. Smooth particle methods represent the fluid as a collection of 'particles' representing macroscopic fluid elements and carrying mass, momentum and thermal energy. The equations of motion governing the smooth particles are derived from the continuum (Navier-Stokes) equations. The pressure and heat flux tensors are determined using linear constitutive relations and an equilibrium equation of state. Specific substances are modelled by the selection of this equation of state and of parameters for the constitutive relations. Lagrangian particle methods bring their own set of numerical challenges for which algorithmic solutions are implemented, including artificial viscosity, anti-clumping measures and the use of different length scales for different components of the equation of state.

The smooth particle equations of motion for a phase separating fluid are solved using parameters derived for water. The condensation of a liquid phase from the water vapour naturally from the solution of the model, with no explicit tracking of the vapour-liquid interface required. Using the smooth particle code to solve the continuum equations of motion for this model of water, we are able to produce droplets and planar gas-liquid interfaces under a variety of boundary conditions. By controlling the mean temperature of the fluid and the volume of the periodic box, the fluid can be taken to arbitrary points on the phase diagram. Expansion may be used to induce boiling, while temperature quenches can be used to produce an instability driven decomposition into coexisting liquid and vapour.

Keywords: SPH, Lagrangian, Condensation 


\section{INTRODUCTION}

\subsection{The van der Waals square gradient model}

The van der Waals equation of state is based on the assumption of a long range mean field attraction between molecules and a hard core repulsion [van der Waals, 1979] [van Kampen, 1964]. It relates equilibrium pressure $p$ with temperature $T$ and has attractive and repulsive parameters $\bar{a}$ and $\bar{b}$. The van der Waals square gradient model describes the inhomogeneous interfacial region as smoothly varying in density. Combining the two we obtain the following for the pressure at a point in a fluid in terms of mass density $\rho$ :

$p(\rho, \mathrm{T})=\frac{\rho \overline{k_{\mathrm{b}}} \mathrm{T}}{1-\rho \overline{\mathrm{b}}}-\rho^{2} \overline{\mathrm{a}}-\bar{M}\left(\rho \nabla^{2} \rho+\frac{1}{2}|\nabla \rho|^{2}\right) \mathbf{1}+\bar{M} \nabla \rho \nabla \rho$

For water, $\overline{\mathrm{a}}=1.045 \times 10^{3} \frac{\mathrm{Jm}^{3}}{\mathrm{~kg}^{2}}, \overline{\mathrm{b}}=1.038 \times 10^{-03} \frac{\mathrm{m}^{3}}{\mathrm{~kg}}, \overline{\mathrm{k}_{\mathrm{b}}}=4.615 \times 10^{2} \frac{\mathrm{m}^{2}}{\mathrm{~s}^{2} \mathrm{~K}}$ and $\bar{M}=9.244 \times 10^{-17} \frac{\mathrm{Jm}^{5}}{\mathrm{~kg}^{2}}$ [Kjelstrup, 2008].

Convenient scaling values are chosen so that numerical solutions are not complicated by extremely large or small numbers, and the equation rewritten in terms of scaled variables. We choose a scaling length of $2.81 \mathrm{~nm}$, a scaling mass of $1.247 \times 10-23 \mathrm{~kg}$, a scaling temperature of $562 \mathrm{~K}$ and a scaling time of one nanosecond. The scaled van der Waals parameters are attraction $\tilde{a}=7.446 \times 10^{4}$, repulsion $\tilde{b}=$ $5.842 \times 10^{1}$ and gradient coefficient $\tilde{M}=8.345 \times 10^{2}$, while the scaled value of Boltzmann's constant is $3.285 \times 10^{4}$.

In the PV plane below the critical temperature the isotherms of the equation of state have two stationary points known as spinodal points and a kink known as a van der Waals loop in which a homogeneous fluid is negatively compressible. The free energy in this region has a double well form which is minimised by an inhomogeneous combination of vapour and liquid states on a constant pressure tangent (the 'Maxwell construction'). The critical temperature is the temperature above which vapour and liquid have the same density. The equilibrium state is the state of minimum free energy $A$. The combination of the constant pressure constraint and common tangent in the PA plane requires the pressure to be minimised subject to $\int_{V_{g}}^{V_{l}} P d V=P_{V}\left(V_{g}-V_{l}\right)$. The binodal curve gives the volumes of coexisting liquid and vapour and is formed by the locus of points minimising $A$ subject to these constraints for isotherms over the range of coexistence temperatures.

A numerical solution for the binodal line was obtained using Brentq optimisation [Brent, 1973], a fast robust root finding method implemented in the scipy package [Scipy]. The pressure satisfying the equal area constraint is found by optimisation of the area function above between the two spinodal pressures. The solution for water is shown in figure 1.

\subsection{A continuum model for condensation}

The continuum equations of motion in the Lagrangian frame are

$$
\begin{aligned}
& \frac{\mathrm{d} \rho}{\mathrm{dt}}=-\rho \nabla \cdot \mathbf{v}, \\
& \frac{\mathrm{d} \mathbf{v}}{\mathrm{dt}}=-\frac{1}{\rho} \nabla \cdot \mathbf{P}, \\
& \frac{\mathrm{du}}{\mathrm{dt}}=\frac{1}{\rho}\left(-\nabla \cdot \mathbf{J}_{\mathrm{q}}-\mathbf{P}^{\top}: \nabla \mathbf{v}\right),
\end{aligned}
$$

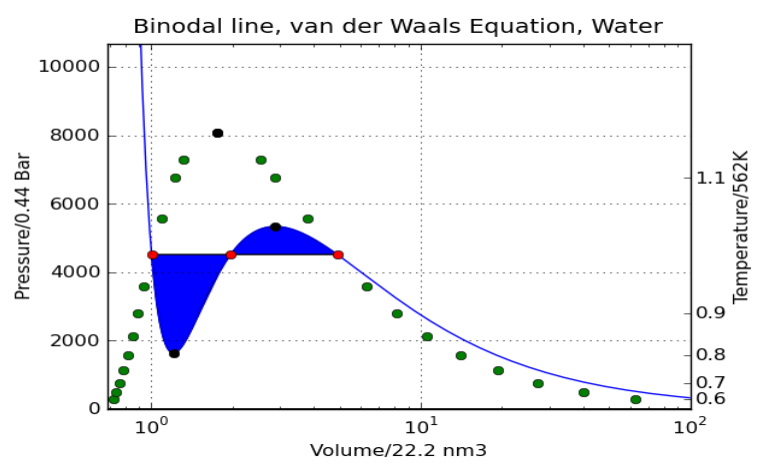

Figure 1: The van der Waals equation of state, with the Maxwell construction tie line, parameterised for water. Green: binodal line, Red: vapour, homogeneous and liquid densities at 562K, Black: spinodal points at $562 \mathrm{~K}$. Blue line: pressure-volume cureve for $\mathrm{T}=562 \mathrm{~K}$. Blue shading: areas equalised by the Maxwell construction. 
with velocity $\mathbf{v}$. The substance specific physics is contained in the pressure tensor $\mathbf{P}$ and the heat flux $\mathbf{J}_{\mathrm{q}}$. We have included contributions from the equilibrium isotropic pressure, Newtonian viscosity with shear and bulk coefficients $\eta$ and $\eta_{v}$ and the the density gradient coefficient $\bar{M}$, where $(\nabla \mathbf{v})^{\text {os }}$ is the symmetric traceless velocity gradient. The full pressure tensor is given by

$\mathbf{P}=\left(\frac{\rho \overline{\mathrm{k}_{\mathrm{b}}} \mathrm{T}}{1-\rho \overline{\mathrm{b}}}-\overline{\mathrm{a}} \rho^{2}\right) \mathbf{1}-2 \eta(\nabla \mathbf{v})^{\mathrm{os}}-\left(\eta_{\nu} \nabla \cdot \mathbf{v}\right) \mathbf{1}-\bar{M}\left(\rho \nabla^{2} \rho+\frac{1}{2}|\nabla \rho|^{2}\right) \mathbf{1}+\bar{M} \nabla \rho \nabla \rho$.

The heat flux is given by Fourier's law $\mathbf{J}_{\mathbf{q}}=-\lambda \nabla \mathrm{T}$. Temperature is related to internal energy $u$ by the equilibrium equation of state $T=\frac{u+\bar{a} \rho}{k_{b}}$ where $\lambda$ is the coefficient of thermal conductivity.

In the solution of this model the vapour-liquid interface is a diffuse region where the density varies smoothly between the bulk liquid and vapour densities.

\subsection{The smooth particle equations}

Mesoscopic, coarse grained models of phase transitions are of interest for their potential to simulate the transient fine scale structure associated with rapid phase transitions and the equilibrium properties of multi-phase systems. Lagrangian methods such as smooth particle hydrodynamics are capable of simulating flows with complex structure naturally. Smooth particle methods represent the fluid as a collection of 'particles' representing macroscopic fluid elements and carrying mass, momentum and thermal energy. Particle arrangements track inhomogeneities in the simulated material for example in the impact frature of a solid [Hoover, 2006] or turbulent magnetohydrodynamics [Price and Federrath, 2010].

A three dimensional smooth particle hydrodynamics code was developed in order to investigate the behaviour of a simple model, parameterised for water, when subjected to temperature and volume quenches into the unstable region of the phase diagram.

The density of each particle is computed as

$\rho\left(\mathbf{r}_{\mathrm{i}}\right)=\sum_{j=1}^{N} m_{j} W\left(\left|\mathbf{r}_{i}-\mathbf{r}_{j}\right|, h\right)$

where $W_{i j}$ is a normalised smoothing kernel. We use Lucy's quartic spline which is computationally cheap and finite in extent. It is given in two dimensions by

$W_{\text {lucy }}=\frac{5}{\pi h^{2}}\left(1+3 \frac{r}{h}\right)\left(1-3 \frac{r}{h}\right)^{3}$

where $h$ is a smoothing length associated with the range of the kernel and $r$ is the distance from the center of the kernel.

The equations of motion governing the smooth particles are derived from the continuum (Navier-Stokes) equations and symmetrised to ensure pair interactions are symmetric. The momentum and energy equations for smooth particles are

$$
\frac{d v_{i}}{d t}=-\sum_{j=1}^{N} m_{j}\left(\frac{P_{s i}}{\rho_{i}^{2}}+\frac{P_{j}}{\rho_{j}^{2}}\right) \cdot \nabla_{i} W_{i j}-\sum_{j=1}^{N} m_{j}\left(\frac{P_{L i}}{\rho_{L i}^{2}}+\frac{P_{L j}}{\rho_{L j}^{2}}\right) \cdot \nabla_{i} W_{L i j}
$$

and

$$
\frac{d u_{i}}{d t}=-\frac{1}{2} \sum_{j=1}^{N} m_{j}\left(\frac{P_{i}}{\rho_{i}^{2}}+\frac{P_{j}}{\rho_{j}^{2}}\right): v_{i j} \nabla_{i} W_{i j}-\sum_{j=1}^{N} m_{j}\left(\frac{J_{q i}}{\rho_{i}^{2}}+\frac{J_{q j}}{\rho_{j}^{2}}\right) \cdot \nabla_{i} W_{i j},
$$

where the summation is over $j$ neighbouring particles, with particle velocity $\boldsymbol{v}_{i}$ and particle internal energy $u_{i}$. Isotropic pressure is given by the van der Waals equation of state using the particle density 
and is separated into short range repulsive and long range attractive components. $\mathbf{P}_{\mathbf{s}}$ represents the short range and $\mathbf{P}_{\mathbf{L}}$ the long range pressure. A long range density is also computed for use in expressions involving the long range force using a longer smoothing length $\mathrm{H}$. To avoid unstable attractive forces we treat the cohesive and repulsive components of the pressure separately, using a longer range for the attractive forces. This is required to obtain well behaved liquid vapour coexistence with SPH. Figure 2 shows interparticle forces at various temperatures and background densities. The left panel shows the interparticle force with $h=2.0$ and $H=4.0$. The right panel shows the interparticle force with $h=H=2.0$. Negative forces are attractive. It is clear from this plot that the $h=H$ case results in uniformly attractive and thus unstable forces between particles for some temperatures and densities. Stability of the simulation requires that interparticle forces become repulsive at a sufficiently high density, close distance. Where a longer ranged kernel is used for part of the force it is used to compute all smoothed properties that go into computing that force. The short range pressure is computed as

$\mathbf{P}_{s i}=\left(\frac{\rho_{s i} \overline{k_{b}} t}{1-\rho_{s i} \bar{b}}\right) 1+-2 \eta \nabla v_{i}^{o s}+-\left(\eta_{v} \nabla \cdot v_{i}\right) 1$

where $\boldsymbol{v}_{i}^{\text {os }}=\frac{1}{2}\left(\nabla \boldsymbol{v}_{i}+\left(\nabla \boldsymbol{v}_{\mathrm{i}}\right)^{\mathrm{T}}\right)-\frac{1}{\mathrm{~d}}\left(\nabla \cdot \boldsymbol{v}_{\mathrm{i}}\right) \boldsymbol{1}$

with velocity gradient

$\nabla v_{i}=\frac{1}{\rho_{i}} \sum_{j=1} m_{j}\left(v_{j}-v_{i}\right) \nabla_{i} W_{i j}$

kernel gradient

$\nabla_{i} W_{i j}=\frac{\mathbf{r}_{i}-\mathbf{r}_{j}}{\left|\mathbf{r}_{j}-\mathbf{r}_{\mathbf{i}}\right|} \frac{\partial W_{i j}}{\partial r_{i j}}$

and velocity divergence

$\nabla \cdot v_{i}=\frac{1}{\rho_{i}} \sum_{j=1} m_{j}\left(v_{j}-v_{i}\right) \cdot \nabla_{i} W_{i j}$

The long range pressure is computed as

$\mathbf{P}_{\mathrm{Li}}=\left(-\overline{\mathrm{a}} \rho_{\mathrm{Li}}^{2}\right)+\bar{M}\left(\rho_{\mathrm{Li}} \nabla_{\mathrm{i}}^{2} \rho_{\mathrm{Li}}+\frac{1}{2}\left|\nabla \rho_{\mathrm{Li}}\right|^{2}\right) \mathbf{1}+M \nabla \rho_{\mathrm{Li}} \nabla \rho_{\mathrm{Li}}$,

where the density gradient is computed using the long range smoothing length as

$\nabla \rho_{i}=\sum_{j=1}^{N} m_{j} \nabla_{i} W_{i j}$

The heat flux $\mathrm{J}_{\mathrm{q}}$ in the energy equation is

$\mathrm{J}_{\mathbf{q} i}=-\lambda\left(\sum_{j} \frac{\mathrm{m}_{i j}}{\rho_{i j}}\left(\mathrm{~T}_{j}-\mathrm{T}_{\mathrm{i}}\right) \nabla_{\mathrm{i}} \mathrm{W}_{\mathrm{ij}}\right)$ 


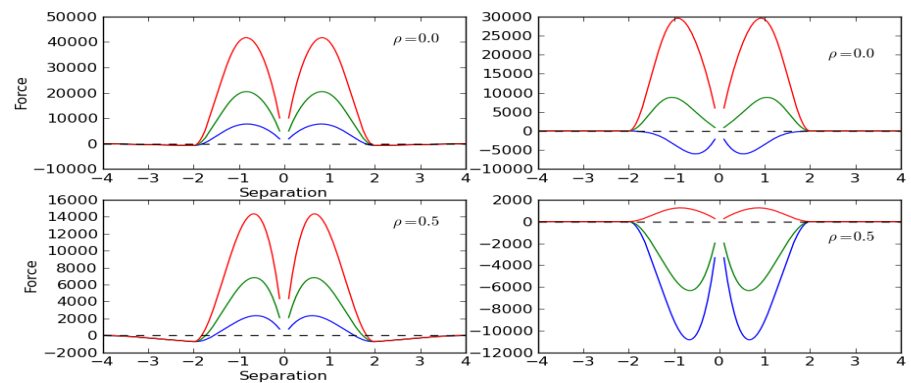

Figure 2: Interparticle force as a function of separation. Left: with long range attraction and short range repulsion. Right: Attractive and repulsive components with the same smoothing length.

\subsection{Liquid Vapour coexistence from 2D volume quenches}

Previously we introduced a 2D implementation of SPH for solving the van der Waals equation of state with the square gradient density approximation. A limitation of this work was that the van der Waals parameters were chosen purely out of convenience, limiting the use of experimental data to validate the theoretical predictions and the simulated results. In this paper we have extended this implementation to solve the equations of motion in three dimensions using a set of parameters derived for water.
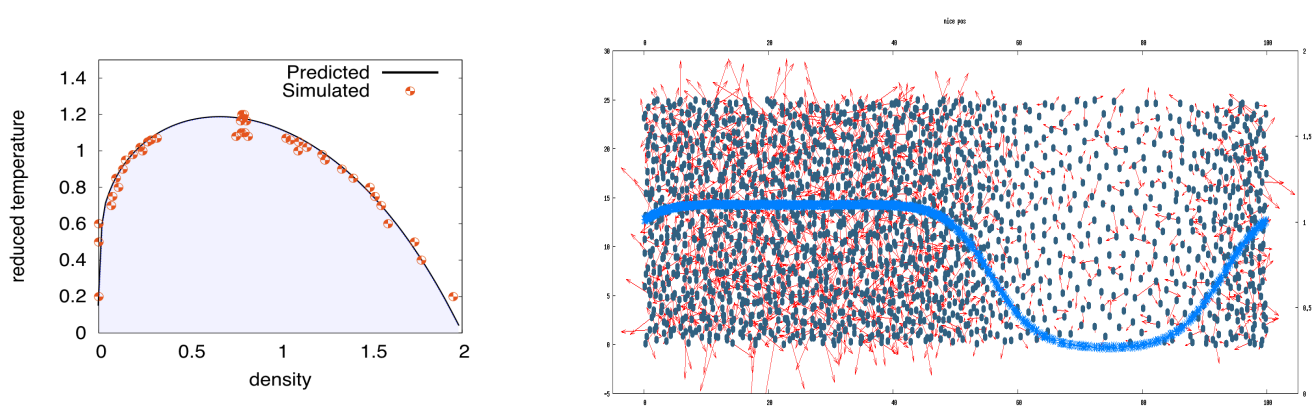

Figure 3: Coexisting densities produced by volume quenches in two dimensions at a range of temperatures. Right: Coexisting densities produced by volume quench in two dimensions at a scaled temperature of 1.05. The blue line is the mean profile density.

In figure 3 we show the results of previous work with a two dimensional smooth particle model initialised in an unstable configuration and allowed to separate into liquid and vapour phases. Following Nugent and Posch [2000], this work showed that the coexisting densities are correctly related to the system temperature. Similar experiments using temperature quenches instead of volume quenches to induce spinodal decomposition in the model system were reported in Charles and Daivis [2009].

\section{COMPUTATIONAL ISSUES}

Particle based methods can be much more computationally intensive than grid based methods. Our model uses a standard Verlet list which is an order $\mathrm{n}^{2}$ neighbour list construction method. Construction of the list is a minor cost, the major cost is the computation of interparticle distances. The next largest computational cost is the string of calculations that must be carried out for each pair. Control code is implemented in python to take advantage of flexibility, while all neighbour list loops and most all particle loops are implemented in Fortran or Cython. Because so much of the computational cost is concentrated in the inner loops over pairs this strategy is possible with little sacrifice of speed. 
To achieve stable simulations of quenches we used a step size of 0.0001 scaling times, achieving simulated times of 0.4 nanoseconds with 4000 timesteps.

The numerical method imposes some stability constraints: certain particle mass settings will disallow the system from reaching certain densities even though these densities are the optimal equilibrium state, while others will allow particles to reach the density threshold $(\rho b=1)$ beyond which interparticle forces are unstable and simulations diverge.

To prevent particles attaining these densities which are not plausible physically and not practical to simulate numerically we impose a hard collision at a distance of 0.6 scaling lengths. It is also necessary to create particles with sufficient mass that the densities desired to be resolved can be attained by particles further apart than this minimum separation. Other means to prevent numerically based degeneration of the simulation include artificial viscosity and artificial repulsive forces.

\section{COMPUTATIONAL EXPERIMENTS}

We first equilibrated a 1000 particle system in a cubic box of a side 10 scaling lengths long where each particle has a mass of 0.4 at a scaled temperature of 1.5. This produced a system with a small range of particle densities given by equation 6 shown in figure 4 .

This is a very small system, of the order of the size of a virus, and smaller by an order of magnitude than engineered nanoparticles ( 200-500nm). Simulation of larger systems could be accomplished by using a larger number of particles, or assigning a larger mass to each particle.

We quenched the vapour to a scaled temperature of 0.5 with parameters described in section 1 for 3000 timesteps with a timestep size of 0.0001 from an initial temperature of 1.5. The simulated quench time is $3.0 \times 10^{-10}$ seconds with the film configuration attained at roughly $5 \times 10^{-11}$ seconds.
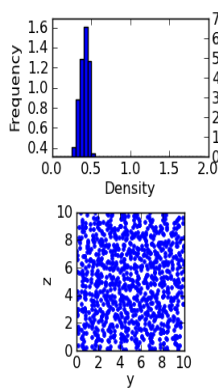
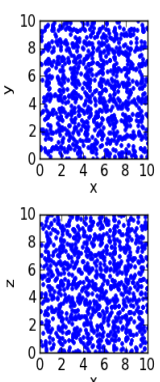
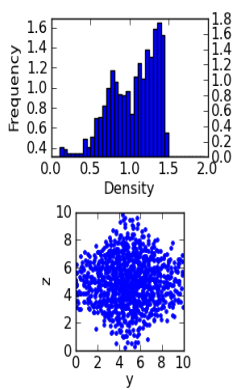
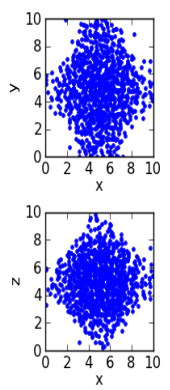
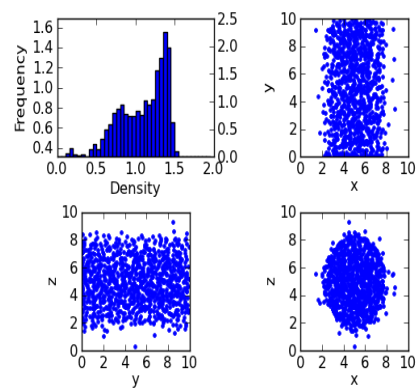

Figure 4: Particle position plot of the quench of a gas to a cylindrical film at $0,1500,2500$ timesteps, with normalised particle density distribution histogram (top left of each frame). Frames show particle positions as seen from the $x y, x z$ and $y z$ planes.

The phase separation of the water develops naturally from the solution of the model, with no explicit tracking of the vapour-liquid interface required. We observe the formation of two distinct phases, although the vapour phase is barely resolved due to the large density difference between vapour and liquid. The liquid phase adopts a curious cylindrical configuration. No particular significance should be attached to this shape other than the observation that it is a minima of the surface area. The periodic boundary conditions used in the simulation stabilise configurations that would not minimise the free energy in unbounded space.

The theoretically predicted liquid density is 1.45 . The maximum particle density is 1.53 . The peak of the density distribution of the final state is between 1.3 and 1.35. This agreement is close enough to be encouraging, although the attained densities are not as a good a fit of expectations as for the two dimensional systems previously studied. Larger simulations are required in order to ensure that a true bulk state has been attained because in these simulations the size of the liquid film is less than the smoothing length. 

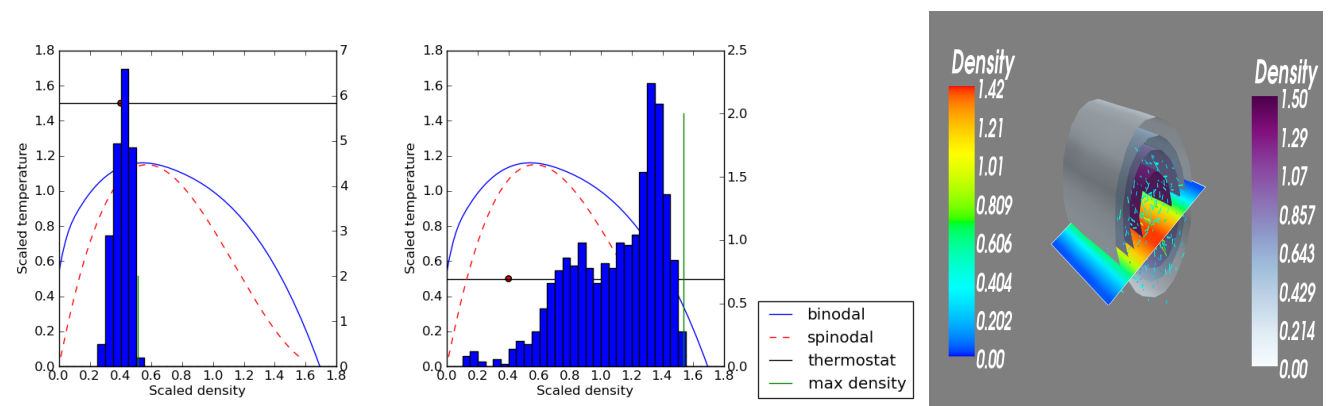

Figure 5: Left: Initial and (middle) final particle density distribution of the quenched formation of a cylindrical film. Right: 3D render with density plane slice and density isosurfaces of final state.

\subsection{Conclusions and Recommendations}

Using the smooth particle code to solve the continuum equations of motion for this model of water we have used a temperature quench to produce an instability driven decomposition into coexisting liquid and vapour with a cylindrical-planar interface.

We have extended our previous modelling of liquid-vapour phase transitions using the simple van der Waals model from two dimensions to three dimensions. We have shown that we can model coexisting phases with densities close to theoretical values for a smooth particle model parameterised for water. Further work using molecular dynamics and experimental studies is needed to determine if the dynamics of phase transitions are captured correctly.

\section{REFERENCES}

Brent, R. P. (1973). Algorithms for Minimization Without Derivatives. Prentice-Hall.

Charles, A. and P. Daivis (2009). Smooth particle methods for vapour liquid coexistence. In 18th World IMACS Congress and MODSIM09 International Congress of Modelling and Simulaton, pp. 303-309. Modelling and Simulation Society of Australia and New Zealand and International Association for Mathematics and Computers in Simulation.

Hoover, W. G. (2006). Smooth Particle Applied Mechanics: The State of the Art (Advanced Series in Nonlinear Dynamics). World Scientific Publishing Company.

Kjelstrup, S. Bedaux, D. (2008). Non-equilibrium Thermodynamics of Heterogeneous Systems. World Scientific Publishing Company.

Nugent, S. and H. A. Posch (2000). Liquid drops and surface tension with smoothed particle applied mechanics. Physical Review E 62(4), 4968.

Price, D. J. and C. Federrath (2010). A comparison between grid and particle methods on the statistics of driven, supersonic, isothermal turbulence. Mon. Not.R. Astron. Soc. 406(3), 16591674.

van der Waals, J. D. (1979, February). The thermodynamic theory of capillarity under the hypothesis of a continuous variation of density. Journal of Statistical Physics 20(2), 200-244.

van Kampen, N. G. (1964, July). Condensation of a classical gas with Long-Range attraction. Physical Review 135(2A), A362.

\section{ACKNOWLEDGEMENTS}

This work was carried out as part of a Commonwealth supported $\mathrm{PhD}$ project at RMIT University in the School of Applied Science, supervised by Professors Peter Daivis and Gary Bryant. Computer time on the Tango cluster was provided by the Victorian Partnership for Advanced Computing (VPAC). 